\title{
DNA module platform for developing colorimetric aptamer sensors
}

\author{
Yasuyuki Tomita', Yuji Morita', Hiroaki Suga², and Daisuke Fujiwara \\ ${ }^{1}$ Central Laboratories for Key Technologies, KIRIN Company, Ltd., Yokohama, Japan and ${ }^{2}$ Department of \\ Chemistry, Graduate School of Science, The University of Tokyo, Tokyo, Japan
}

BioTechniques 60:285-292 (June 2016) doi 10.2144/000114425

Keywords: DNA module platform; aptamer-based sensor; aptamer microarray; in silico design

Supplementary material for this article is available at www.BioTechniques.com/article/114425.

Here we present a DNA module platform for developing simple aptamer sensors based on a microarray format combined with secondary structure prediction in silico. The platform comprises four parts: (i) aptamer, (ii) joint module, (iii) terminal stem, and (iv) a DNAzyme that catalyzes a redox reaction controlled by a structural change induced by aptamer/target binding. First, we developed a joint module, capable of sensing a conformational change in the aptamer region, that was linked to the signal transmission activity of a DNAzyme as the reporter in a concentration-dependent manner with the AMP aptamer. This module design was then used to generate an arginine sensor simply by replacing the AMP aptamer region with a previously reported arginine aptamer. Using this DNA module platform, we were also able to customize a microarray containing $>10,000$ sequences designed by in silico secondary structure prediction and successfully identify a new aptamer against patulin. Our results show that the DNA module platform can be used to readily devise sensors based on known aptamers as well as create new aptamer sensors by array-based screening.

Aptamers offer several advantages over traditional antibodies, including ease of synthesis and modification, high stability and low cost. Aptamers can be also applied to electrochemical (1-4), colorimetric $(5,6)$, fluorescence-based optical (7), and mass-sensitive methods $(8,9)$. These features render aptamers wellsuited to real-time, on-site analyses, such as those required for production management of industrial products including foods and beverages.

Existing platforms for aptamer sensor development often require two steps: (i) isolation of aptamers against the target of interest from a pool of RNA or DNA molecules and (ii) development of an aptamer-based sensor from the appropriate aptamer (10). To isolate desired aptamers, a selection technique known as SELEX (systematic evolution of ligands by exponential enrichment) is generally used (11,12). However, SELEX requires technical expertise because it involves large sequences libraries and complicated selection operations. To develop an aptamer-based sensor for practical analysis, it is necessary to optimize the detection process for a target molecule by combining an isolated aptamer and an appropriate method.

Recently, designed aptamers have been reported whose conformational change upon binding to a target molecule could be applied in aptamerbased sensing process. Target analyteinduced conformational changes were converted into signals, such as colorimetric, fluorescence-based, or electrochemical signals by a DNAzymebased redox reaction $(13,14)$, disas- sembly of gold nanoparticle aggregates $(15,16)$, detection of captured aptamers by capture DNA immobilized onto a support (17), or cleavage by DNAzymes $(10,18)$. These sensor platforms, however, have never been applied to screening of aptamers or optimization of the sequences relevant to conformational changes.

Here we present a DNA module platform for designing simple aptamer sensors based on a microarray format combined with the secondary structure prediction in silico. Our platform comprises four regions; aptamer, joint module, terminal stem, and a DNAzyme comprising a horseradish peroxidase (HRP)-mimicking DNAzyme (13) that catalyzes a redox reaction controlled by a structural change induced by binding of the aptamer to its target.

\section{METHOD SUMMARY}

Here, we present a new methodology for generating aptamer-based sensors based on a newly developed DNA module platform comprising four parts; aptamer, joint module, terminal stem, and signal region. To construct an aptamer sensor, the following operations are performed: fusing the regions, modifying region sequences in silico, and optimizing by microarray. 


\section{Materials and methods}

Joint module design and optimization

First, an aptamer sensor based on an allosteric DNAzyme was designed as shown in Figure 1. Aptamer (AR), signal $(\mathrm{SR})$, and terminal (TR) regions were linked through a joint module (JM). The JM is subdivided into two regions: an aptamermasking region and a linker region. The JM transmits the effect of a conformational change following binding of the AR to its target SR. The design of the $J M$ is crucial for efficient and effective transmission of this signal. Aiming at optimizing the JM, we fused a DNAzyme sequence that mimics HRP (13) to a previously described AMP aptamer (19); this design should produce a system where the oxidation/reduction reaction can be detected as an electrochemical signal monitored by a microarray chip, specifically the CombiMatrix 12K ElectraSense chip (20). Each chip contains 12,544 spots, and a total of 544 spots were used for fabrication and quality control, thus allowing simultaneous evaluation of 12,000 possible JM sequences. These customized ElectraSense microarrays were purchased from Recenttec K. K. (Tokyo, Japan).

The following prerequisites were essential for designing an appropriate JM: the length of the aptamer-masking region should be three or four bases, the linker region length should range from one to five bases, and internal and bulge loops should be present in the aptamer-masking region and an internal loop in linker region. From an extensive potential pool of JM sequences, we selected 2720 candidates containing objective 2-D hairpin structures that were predicted in silico (Figure 1A). The DNA structure was predicted using hybrid-ss-min (UNAFold) version 3.8 software (http://mfold.rna.albany.edu) (21) using the DNA input mode with settings of $20^{\circ} \mathrm{C}$ and $0.2 \mathrm{M} \mathrm{NaCl}$ (equivalent to the binding conditions used in the in vitro assays). Each of the 2720 candidate JMs was fused between the AMP aptamer (19) and the DNAzyme (13), and we subsequently analyzed the efficiency of transmission of the $\mathrm{ON}$ signal from the AR to the SR as described below.

\section{Electrochemical and colorimetric} detection of DNAzyme activity

The oxidation-reduction reaction activity of the DNAzyme was evaluated by comparing the electrochemical signal generated in the presence of the DNAzyme to that generated by a non-heme binding DNA oligomer (13) using the microarray technology in accordance with the following protocol. Both DNAzyme and control sequences were linked to poly-dT (15) at their 3' ends and synthesized directly onto five independent electrodes of the microarray chip. The microarray chip was immersed in $120 \mu \mathrm{L}$ buffer [25 mM HEPES, 200 mM NaCl, $20 \mathrm{mM} \mathrm{KCl}$, and $0.1 \%$ (v/v) DMSO, pH 7.4] and incubated for $10 \mathrm{~min}$ at $65^{\circ} \mathrm{C}$. The chip was then cooled slowly to room temperature over $30 \mathrm{~min}$ in order to allow the probes to fold into their correct conformations. Then, hemin solution was added (2.5 $\mu \mathrm{M}$ hemin final concentration in the buffer), and the chip was incubated for 15 min at room temperature. Finally, the solution was replaced with the substrate solution [5 mM 2,2'-azino-bis-(3-ethylbenzthiazoline-6-sulfonic acid) (ABTS) and 5 $\mathrm{mM} \mathrm{H}_{2} \mathrm{O}_{2}$ in the solution] and mixed gently by repeated pipetting before scanning the microarrays. The electrochemical reactions on the microarray were detected using an ElectraSense Reader (CombiMatrix, Irvine, CA) in accordance with the manufacture's protocol. Since the electrochemical signal from the ElectraSense remained unchanged for at least 5 min when ABTS was used as an electron donor (data not shown), the signal was measured 1 min after addition of substrate for all subsequent experiments.

Next, each of the 2720 candidate fusion sequences for the SR, JM, AR, and TR (CCC) (Figure 1A) was linked to poly-dT (15) at the 3 ' end and synthesized directly onto the electrodes of a microarray chip. The following operation was performed after the chip was cooled to room temperature over 30 min: The buffer was replaced (containing no or 5 $\mathrm{mM}$ AMP), and the chip was incubated for 30 min at room temperature. Evaluation of each of the JMs was carried out by normalizing the signal obtained in the presence of $5 \mathrm{mM}$ AMP to that in the absence of AMP. Each sequence was spotted as triplicates or quintuplets. Experiments were repeated twice and correlated. In addition, we compared the results with those obtained with the JM reported by Teller et al. (22), referred to herein as "original JM," which forms a perfectly complementary joint sequence (Figure 2).

To achieve our goal of developing a label-free aptamer sensor that makes

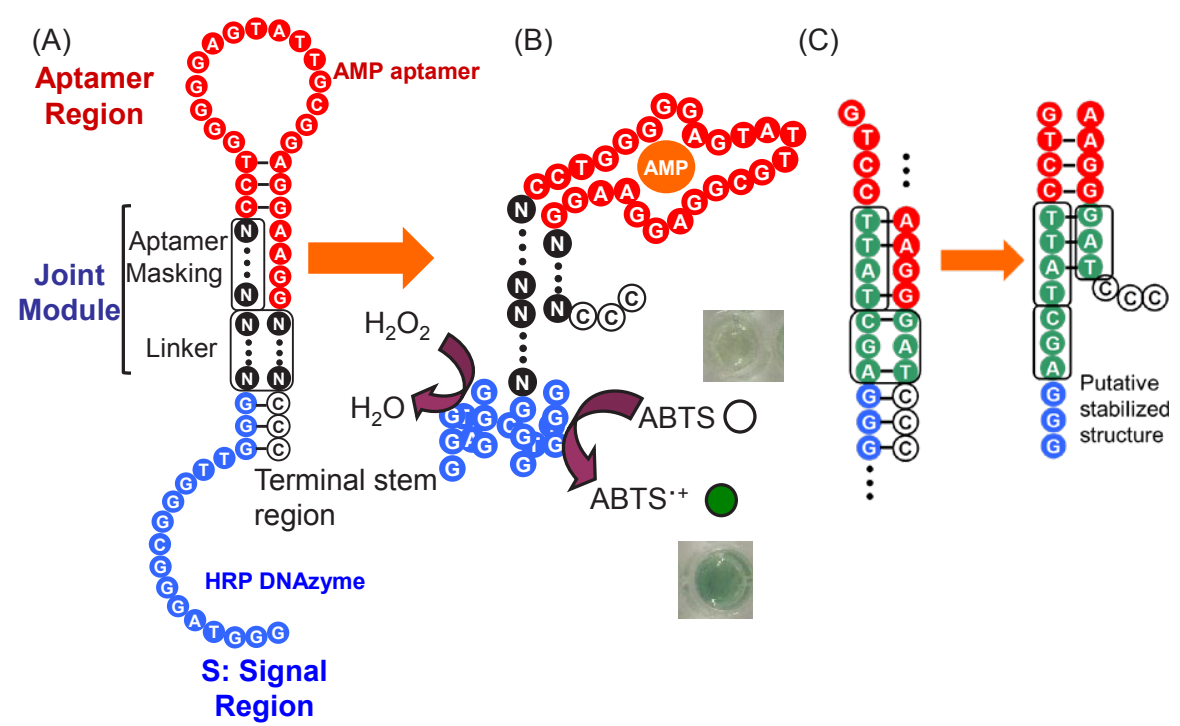

Figure 1. Detection scheme of the DNA module platform. (A) Structural design of aptamer sensors used in this study. (B) Colorimetric detection of aptamer binding was monitored by using a horseradish peroxidase (HRP)-mimicking DNAzyme as the signal region, which converts 3-ethylbenzthiazoline-6-sulfonic acid (ABTS) into a green chromophore that can be detected by the absorbance change. To improve sensitivity, a modified joint module (JM) was designed that integrates the aptamer (AR) and signal (SR) regions. An AMP aptamer was used to optimize the JM. In addition, the reaction could also be detected by monitoring the electrochemical signal arising from the redox reaction. (C) The putative mechanism of the aptamer sensor described in this study. Before sensing the target molecule (left), the 3' terminus of the AR is masked by the aptamer-masking region. After binding the target molecule (right), the masking sequence may enhance the stability of the ligandbound conformation of the aptamer sensor by forming new base pairs with one of the linker regions. 
use of a simplified colorimetric detection system, we confirmed the activity of the isolated JMs by measuring the absorbance change induced by the presence of the associated sensors in solution as shown in Figure 1. DNA constructs were purchased from Life Technologies (Tokyo, Japan). All oligonucleotides were cartridge-purified and freeze-dried by the company and diluted in $10 \mathrm{mM}$ HEPES with $10 \mathrm{mM} \mathrm{NaCl}$ to generate stock solutions of $100 \mu \mathrm{M}$. Experiments were performed in $25 \mathrm{mM}$ HEPES buffer with $200 \mathrm{mM} \mathrm{NaCl}, 20 \mathrm{mM} \mathrm{KCl}$, and $0.1 \%$ (v/v) DMSO, $\mathrm{pH}$ 7.4. DNA was diluted to a concentration of $12.5 \mu \mathrm{M}$ with buffer, heated to $90^{\circ} \mathrm{C}$ for $5 \mathrm{~min}$, and slowly cooled to room temperature over 30 min. Ligand was added and allowed to interact with the DNA for $30 \mathrm{~min}$. Hemin and ABTS were added to a final concentration of $0.5 \mu \mathrm{M}$ and $5 \mathrm{mM}$, respectively. The reaction was initiated by addition of hydrogen peroxide (final concentration 5 $\mathrm{mM})$. Color development was followed at $414 \mathrm{~nm}$ with a BioTek EON Microplate Spectrophotometer. The oxidation absorbance changes (the ratio of $A_{414, x}$ ) at ligand concentration $x$ compared with buffer alone due to the hemin-catalyzed oxidation of ABTS- by $\mathrm{H}_{2} \mathrm{O}_{2}$ were calculated using the following equation at 5 min after addition of $\mathrm{H}_{2} \mathrm{O}_{2}$ and averaged over 3 trials: Ratio of $A_{414, x}=A_{414, x} / \bar{A}_{414,0}$. Furthermore, in order to ascertain whether the isolated JM bears universality, an arginine aptamer (23) was also tested as the AR.

Rules for design of aptamer candidates We synthesized 12,000 different candidate AR sequences comprising 30 bases fused to Joint 5, the DNAzyme, and the TR (CCC). AR candidates were designed in silico using the following criteria: (i) the predicted 2-D structure in the absence of ligand forms one hairpin loop; (ii) the length of the hairpin loop comprises three to seven nucleotides; and (iii) the length of the stem comprises six to nine nucleotides. Finally, 12,000 candidates were randomly selected from 14,441 candidates with a 2-D hairpin structure predicted by the in silico analysis, because of the limitation of the number of samples available on the microarray plate. Detailed information on the design is shown below and in Supplementary Figure S1. Each sequence was linked to poly-dT (15) at the 3'-end and synthesized directly onto two

Figure 2

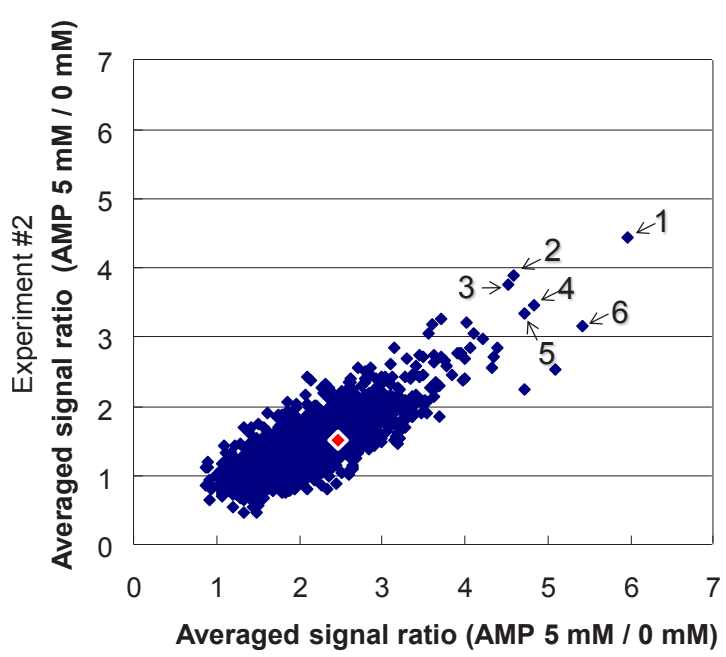

Experiment \# 1
Joint module sequence

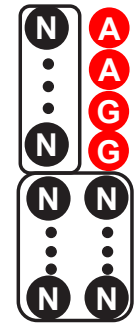

2,720 designed joints

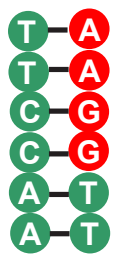

Original joint module

Figure 2. Scatter blot of signals obtained from the aptamer array for optimizing a more efficient joint module (JM). The 2720 designed JMs containing a variety of bulges and internal loops. Microarray technology was applied to evaluate the efficiency of the JMs by comparing them with a JM reported by Teller et al. (22), which forms a perfectly complementary chain, referred to here as the "original joint module." The efficiency was evaluated by normalizing the signal obtained in the presence of 5 $\mathrm{mM}$ AMP to that obtained in the presence of buffer alone. Experiments were repeated twice, and the results were correlated between the two experiments. The red and blue plotted points represent the normalized signals from the original JM and the designed JMs, respectively. Numbers (1-6) indicate selected JMs whose signals were superior to that of the original JM and were therefore studied further.

independent electrodes of the microarray chip; that is, each sequence was spotted as a doublet.

Screening of candidates for the patulin aptamer using microarray technology

Screening of a patulin aptamer was carried out by a method similar to that used for JM isolation. The following operation was performed after cooling the chip to room temperature for 30 min: The buffer was replaced (containing no or 100 $\mu \mathrm{M}$ patulin), and the chip was incubated for $30 \mathrm{~min}$ at room temperature. Evaluation of each candidate was done by normalizing the signal obtained in the presence of the analyte to that obtained with buffer alone. Thirty patulin aptamer candidates were screened that displayed a signal in the presence of patulin that was 1.5-fold greater than the control for each of the doublet probes. We subsequently evaluated the aptamer candidates using the colorimetric assay to assess dosedependency and sensitivity of detection of patulin. Furthermore, with respect to a patulin aptamer, we examined the specificity using two molecules, benzofuran, and (S)-patulin methyl ether, which have structures similar to that of patulin. Finally, in order to improve the sensitivity of the patulin aptamer-sensor, we examined its activity in the context of three types of TR (CCC, CCCA, and CCCAA).

\section{Results and discussion}

The redox reaction activity of the DNAzyme was first evaluated by monitoring the electrochemical signal using a microarray format and comparing it to that to a non-heme binding DNA oligomer (13). The microarray system enables detection of the redox reaction of the HRP-mimicking DNAzyme using ABTS and $\mathrm{H}_{2} \mathrm{O}_{2}$ substrates (Supplementary Figure S2), leading to green color development in the solution. Because the activity of the DNAzyme was readily detected as an electrochemical signal in the microarray format, we next optimized the JM.

We examined whether the JM was able to effectively sense a conformational change of the reporter AMP aptamer region, enabling detection of signals from the DNAzyme in an AMP concentrationdependent manner. We measured the ratio of the signal developed in the presence of 
$5 \mathrm{mM}$ AMP to that developed with buffer alone. Results obtained for each of the 2720 candidate sequences comprising the SR (HRP-mimicking DNAzyme), the library of candidate JMs, the AR, and the TR (CCC) were then compared with a sequence including the original JM as a control (Figure 2). We observed a good correlation between the results of 2 independent experiments (correlation factor $=0.799$ ), leading to the identification of a number of candidate JMs from the library displaying higher sensitivity than the original JM. Because the differences in the signal ratio depended on the JMs, we evaluated the tendency to display greater electrochemical signal ratio according to seven parameters: (i) free energy of the secondary structure, (ii) length of the aptamer-masking (AM) region, (iii) the number of internal loops in the AM, (iv) the number of bulge loops in the AM, $(v)$ the length of the linker region, (vi) the number of internal loops in the linker, and (vii) the GC content of the linker (Supplementary Figure S3). For example, although effective JMs tend to have free energies in the range of -10 to $-6 \mathrm{kcal} / \mathrm{mol}$ in the absence of ligand, this is not sufficient to lead to high sensitivity as the other 6 parameters are similar to the pattern of free energies and can contribute to the sensitivity. Among the JMs that were more effective than the original JM, six were selected for further study and were referred to as Joint 1 through 6.

Next, we reproduced the microarray results using the colorimetric assay with sensors containing Joints 1 through 6 . Joints 1, 5, and 6 showed a dosedependent increase in absorbance in the presence of the ligand (Figure 3). Joint 5 (Sequence \#1 in Table 1) displayed the greatest absorbance change at lower concentrations of AMP and minimumvariance at $0 \mu \mathrm{M}$ (Figure 3). For example, absorbance changes at 50 and $500 \mu \mathrm{M}$ in Joint 5 were 0.076 and 0.15 , respectively. On the other hand, absorbance changes at 50 and $500 \mu \mathrm{M}$ in the Original Joint were 0.022 and 0.084 , respectively (Supplementary Figure S4). Thus, there was an improvement in sensitivity of AMP detection with our newly optimized joint module. Interestingly, Joints 1, 5, and 6 shared a consensus sequence within the aptamer masking region (AM) (TRTT) in the $5^{\prime}$ to $3^{\prime}$ direction, in which the $5^{\prime} \mathrm{T}$ forms a mismatch pair with the $G$ at the
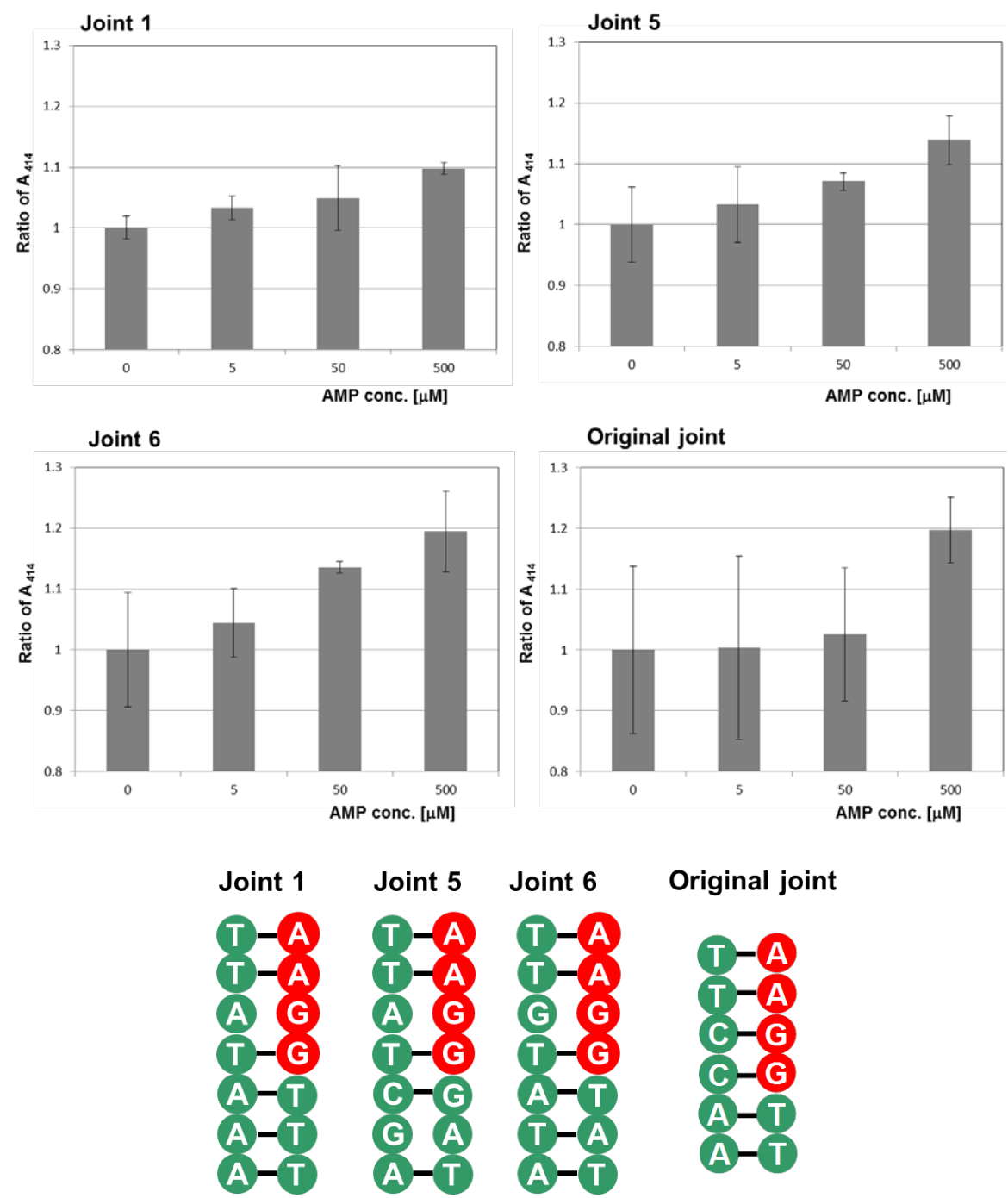

Figure 3. Colorimetric assay using aptamer sensors with Joints $1,5,6$, and the original Joint Module (JM). The $y$-axis represents the absorbance change (see equation the "Materials and methods" section) at the concentrations shown on the x-axis.

$3^{\prime}$ end of the AR; where "R" indicates an A or $G$ that forms an internal loop, and the two 3' Ts pair with As at the 5' side of the masked AR region. This sequence conservation suggests these elements might be important for the enhanced activity.

We then examined if this module design could be used to generate an arginine sensor by simply replacing the AMP aptamer region with a known arginine aptamer (23). The aptamermasking region was four bases long

and contained an internal loop, whereas the linker region was identical to that of Joint 5 (Sequence \#2 in Table 1; Figure 4). The AM was modified from Joint 5 in AMP sensor to reflect the 3' terminal sequence of the arginine aptamer. In addition, we compared Joint 5 with a JM that formed regions of perfect sequence complementary with lengths of four and two bases in the AM and linker regions, respectively, as well as with the original $\mathrm{JM}$ in the AMP aptamer sensor (Figure 4) (22). Compared with the original JM,

Table 1. Sequences of the oligonucleotides (in the $5^{\prime} \rightarrow 3^{\prime}$ direction) used in this study.

\begin{tabular}{|lll|}
\hline ID & Ligand & \multicolumn{1}{c|}{ Sequence } \\
\hline Sequence \#1 & AMP & GGGTAGGGCGGGTTGGGAGCTATTCCTGGGGGAGTATTGCGGAGGAAGGGATCCC \\
Sequence \#2 & Arginine & GGGTAGGGCGGGTTGGGAGCGACGACCAGGGCAAACGGTAGGTGAGTGGTCGATCCC \\
Sequence \#3 & Patulin & GGGTAGGGCGGGTTGGGAGCTATTCCTGCGGGCGCTGTTCGCCTAGTCGGAAGGGATCCC \\
\hline asignal region (SR) (in italics); joint module (JM) (in bold); aptamer region (AR) (underlined). \\
\hline
\end{tabular}


Joint 5 conveyed enhanced sensitivity to the arginine aptamer (Figure 4). Therefore, we conclude that Joint 5 appears to be a general enhancer sequence for aptamer sensors.

Patulin, with a molecular weight of only $154 \mathrm{~g} / \mathrm{mol}$, is a mycotoxin produced by fungal species that frequently grow on fruit and vegetables.. To date, a useful biosensor based on an aptamer for patulin has never been reported. In order to perform the general SELEX protocol, patulin must be chemically modified for immobilization. However, because of its rather simple molecular structure, it is difficult to design an appropriate patulin analog without altering the chemical structure $(24,25)$. This might have hindered the development of aptamer sensor against patulin. We theorized that our aptamer microarray system would be an appropriate method for the development of an anti-patulin aptamer sensor.

ARs were designed in silico according to the following criteria: (i) presence of a predicted 2-D structure forming one hairpin loop in the absence of ligand; (ii) hairpin loop length of 3-7 nucleotides, and (iii) stem length of 6-9 nucleotides. The number of exhaustive combinations of dot-brackets indicating secondary structure was calculated under the abovementioned three conditions $(873,694$ dot-bracket combinations, Supplementary Figure S1). In order to select allowable bracket and dot combinations, GC pairs contributing the most stable free energy and $A$ residues unpaired with $G$ and $C$ were assigned to bracket and dot sites, respectively. The secondary structures of 873,694 sequences were then predicted. We found 3,96 sequences to be consistent with their bracket and dot combinations. A, T, G, and C were assigned to these 3896 secondary structures in the following manner. In each secondary structure, 1000 unique sequences were generated under the following 2 conditions: (i) GC, AT, and TG pairs were randomly assigned to the stack regions, but the GC pair was preferentially assigned; (ii) The bases in loop regions were randomly assigned with equivalent probabilities of generating $A$, $\mathrm{T}, \mathrm{G}$, and $\mathrm{C}$. The secondary structures of the 3,896,000 unique sequences were predicted, and 171,530 different sequences were selected according to their agreement with the structural rules outlined above. As a result, the ratio of GC, AT, and TG pairs in the stack regions was 86:12:2, respectively. Finally, each candidate was combined with the SR (DNAzyme), Joint 5, and the TR (CCC), and then their secondary structure was predicted. Candidates with the objective 2-D hairpin structures (Figure $1 \mathrm{~A})$ were retained as the final candidates $(14,111$ total sequences). Because only 12,000 candidates could be simultane-

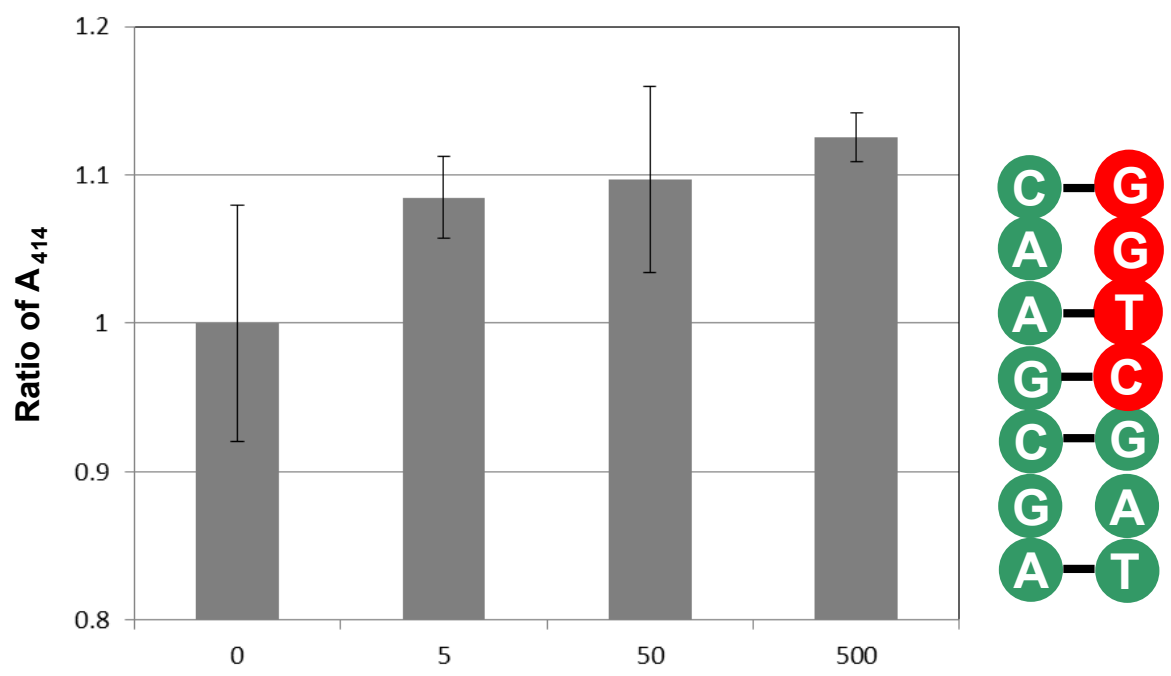

Arginine conc. $[\mu \mathrm{M}]$

Figure 4. Colorimetric assay to compare the sensitivity of detection of arginine with an aptamer sensors incorporating Joint $\mathbf{5}$ selected from the screen in Figure 2. The aptamer-masking region (AM) was four bases long and contained one internal loop, whereas the linker region was identical to that of Joint 5 (Sequence \#2 in Table 1). The y-axis represents absorbance change see equation the "Materials and methods" section) at the concentrations shown on the $x$-axis. Joint 5 and the original joint module (JM) are shown. ously evaluated, 2,111 candidates were removed by excluding candidates with a hairpin loop length of 6 or 7 and those with a stem site length of 9 nucleotides.

We selected 30 patulin aptamer candidates that exhibited a normalized signal ratio of 1.5-fold or greater (Supplementary Figure S5). The reproducibility of the observed sensitivity in the selected candidates was confirmed by monitoring the absorbance change in the colorimetric assay. We then selected 3 candidates showing signals that correlated with increases in patulin concentration $(0,0.005,0.05,0.25,0.5$, and 2.5 $\mathrm{mM}$ ) (Supplementary Figure S6). Among the latter, Candidate 3 could detect 50 $\mu \mathrm{M}$ patulin with an absorbance change of 0.05. In order to evaluate the specificity of candidate 3 to patulin, two structurally similar molecules [benzofuran, and (S)-patulin methyl ether] were tested. As shown in Figure 5, Candidate 3 gave rise to an increased absorbance change in a concentration-dependent manner against patulin, but not when tested in the presence of either of the two structurally similar molecules.

In order to further improve the sensitivity of Candidate 3 (Sequence \#3 in Table 1), we examined 3 types of TRs (CCC, CCCA, and CCCAA) at lower concentrations of patulin $(0,1,5,10$, and $25 \mu \mathrm{M})$. Among the TRs tested, CCCA was shown to improve the sensitivity of patulin detection with a 0.1 absorbance change at a patulin concentration of 25 $\mu \mathrm{M}$ (Supplementary Figure S7). Thus, we have succeeded in further improving the sensitivity by customizing the TR, a part of our DNA module platform.

Here we developed a DNA module platform for designing simple aptamer sensors based on a microarray format combined with the secondary structure prediction in silico. We have found that the joint module, one of four regions in the DNA module platform, was a key component for sensitivity and succeeded in screening for effective JMs that were able to sense conformational changes in the reporter AMP aptamer region linking to the signal transmission activity of the DNAzyme in a concentrationdependent manner. We also showed that this module design was capable of generating an arginine sensor simply by replacing the AMP aptamer region with a reported arginine aptamer. Furthermore, 

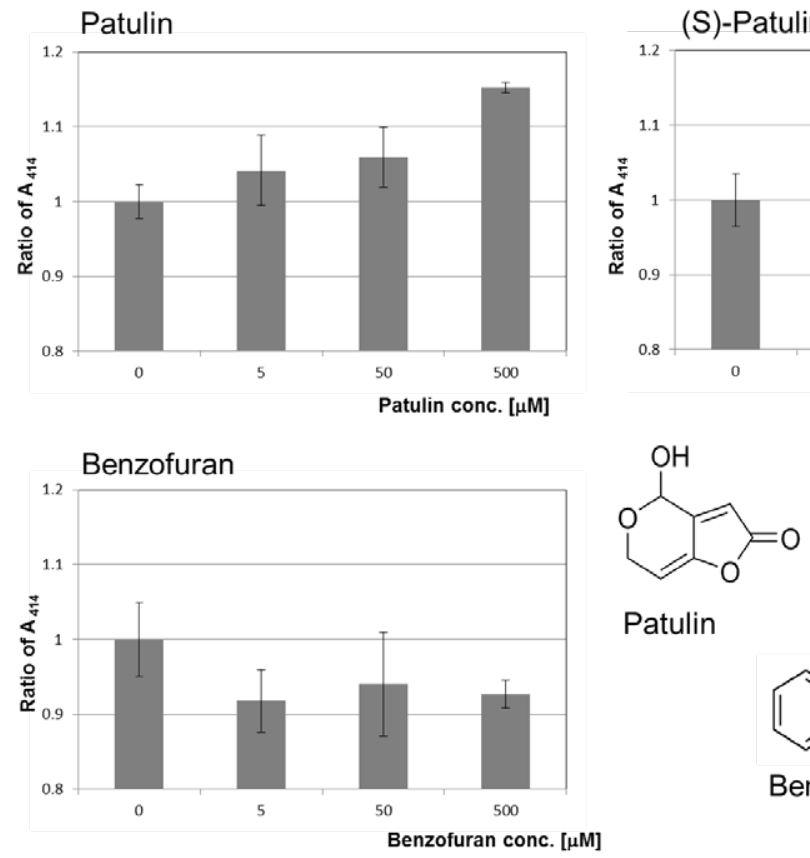

Patulin

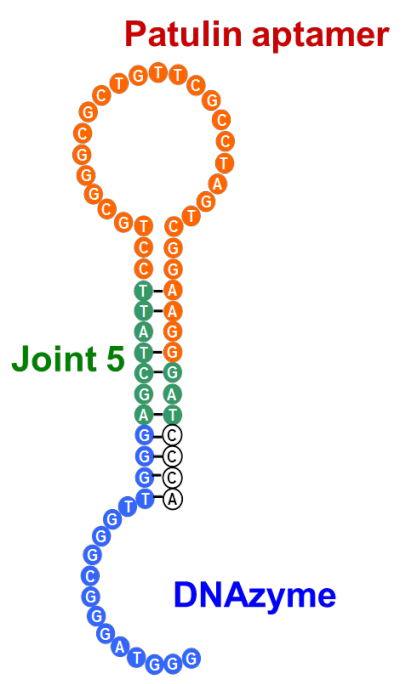<smiles>c1ccc2occc2c1</smiles>

Benzofuran

TAKE ACTION AGAINST

\section{CONTAMINATION WITH \\ QMI $^{\circledR}$ SAFE-SEPTUM}

Sample, Inoculate Or Add Nutrients

To Your Bioreactor Aseptically!

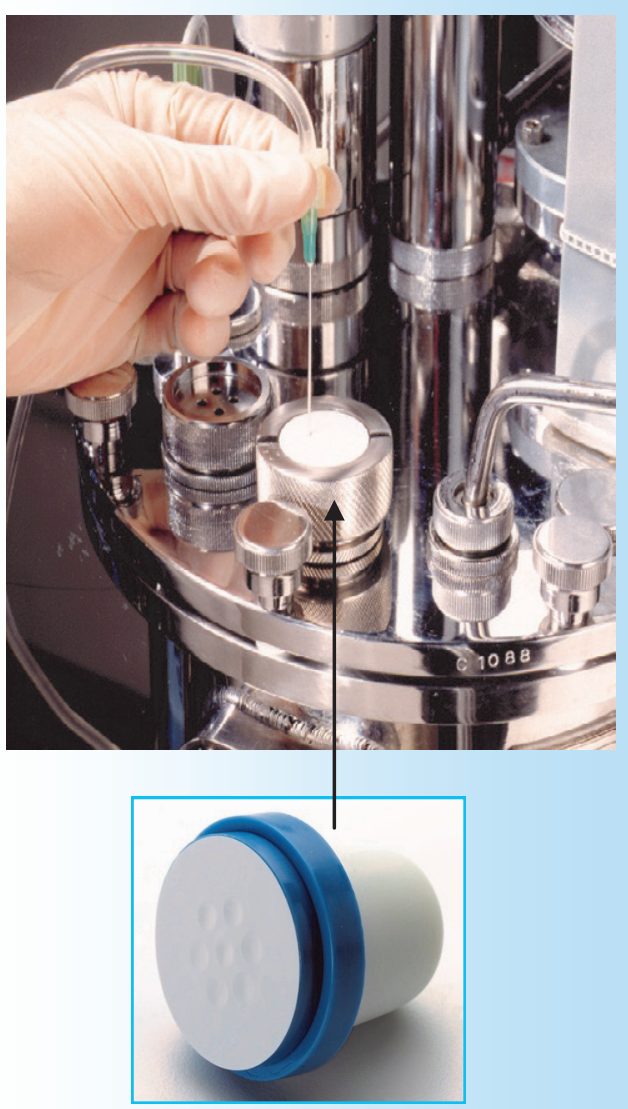

The QMI Safe-Septum is:

Figure 5. Colorimetric assay using the patulin aptamer sensor developed in this study. The ligand specificities of the selected patulin aptamer were investigated by using two similar analyte molecules: benzofuran and (S)-patulin methyl ether. The y-axis represents absorbance change (see equation the "Materials and methods" section) at the concentrations shown on the x-axis.

this DNA module platform was applied to an aptamer array screening system, and aptamers that recognized patulin were obtained by the system.

In our JM-modified DNA module platform, Joint 5 appears to effectively enhance the conformational change of the aptamer sensor by transmitting the energy acquired by target molecule binding efficiently and rapidly to the DNAzyme. The complementary AM sequence in Joint 5 may enhance the stability of the entire aptamer sensor structure, leading to inactivation of aptamer binding structure and also the binding to the target molecule by base pairing with one of the linker regions as shown in Figure $1 \mathrm{C}$. In addition, the enhanced activity of Joint 5 was found to be independent of the sequence of the $\mathrm{AR}$ and other peripheral regions in the arginine aptamer sensor. These features would allow us not only to generate an aptamer-sensor simply by replacing the AR with a known or screened aptamer, but also to develop simple aptamer sensors based on a microarray format combined with secondary structure prediction in silico. Moreover, this microarray system would also enable us to increase sensor sensitivity by systematically optimizing
- Aseptic

- Pressure \& Temperature Safe

- Pre-Sterilized

- Easy To Retrofit

- Validated

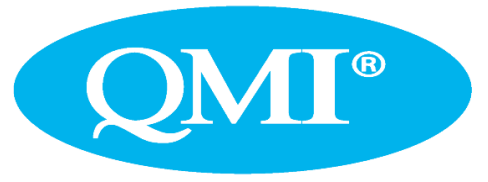

651-501-2337

Email: info@qmisystems.com 
individual sensor regions (AR, SR, TR, and JM) by testing simultaneously the effects of introduction of arbitrary sequences into specific regions in an iterated fashion that allows repeated stages of optimization.

Among seven positive patulin aptamer candidates, we identified a sequence that detected patulin with high sensitivity and reproducibility. Unfortunately, the present patulin aptamer sensor did not fully meet the minimum sensitivity requirement for screening patulin in foods and beverages suggested by WHO (26), and thus it needs to be improved for higher sensitivity. This might require further optimization of the sensor itself to improve the sensitivity, or appropriate enrichment of the patulin concentration during sample preparation. However, conventional detection methods for patulin, such as thin-layer chromatography (TLC) (27) or high-performance liquid chromatography-mass spectrometry (HPLC-MS) (28), are either insufficiently sensitive or require the use of an expensive instrument with sophisticated handling that is not convenient for real-time and on-site detection. In contrast, aptamer sensors, albeit requiring some improvement, potentially allow us to detect target molecules by real-time and on-site since they requires only a simple colorimetric detection of the green color by the naked eye.

Even though the size of an aptamer microarray library $\left(10^{4}\right)$ is much smaller than that normally required by the SELEX method $\left(\sim 10^{15}\right.$, covering the entire range of 25 bases), we were able to identify aptamers against small molecules using our DNA module platform based on a microarray format combined with secondary structure prediction in silico. Importantly, the platform proved less timeconsuming and labor-intensive compared with existing methodologies, allowing for development of a colorimetric detection system for real-time and on-site detection of target molecules.

\section{Author contributions}

Y.T. conceived the study and designed the experiments. Y.T. and Y.M. performed the experiments. Y.T. designed sequences in silico and analyzed the data. H.S. performed technical consultation on planning experiments. D.F. supervised this project. Y.T. wrote the paper, which was edited by all of the authors.

\section{Acknowledgments}

We are grateful to members of Central Laboratories for Key Technologies, KIRIN Company, Ltd, especially to Satoru Mizutani and Aruto Yoshida for their helpful discussion and to Taeko Akiyoshi for her technical assistance.

\section{Competing interests}

The authors declare no competing interests

\section{References}

1. Xu, D., D. Xu, X. Yu, Z. Liu, W. He, and Z. Ma 2005. Label-free electrochemical detection for aptamer-based array electrodes. Anal. Chem. 77:5107-5113.

2. Numnuam, A., K.Y. Chumbimuni-Torres, Y. Xiang, R. Bash, P. Thavarungkul, P. Kanatharana, E. Pretsch, J. Wang, and E. Bakker. 2008. Aptamer-based potentiometric measurements of proteins using ion-selective microelectrodes. Anal. Chem. 80:707-712.

3. Wang, X., J. Zhou, W. Yun, S. Xiao, Z. Chang, P. $\mathrm{He}$, and Y. Fang. 2007. Detection of thrombin using electrogenerated chemiluminescence based on $\mathrm{Ru}(\mathrm{bpy}) 3(2+)$-doped silica nanoparticle aptasensor via target protein-induced strand displacement. Anal. Chim. Acta 598:242-248.

4. Ikebukuro, K., C. Kiyohara, and K. Sode. 2005. Novel electrochemical sensor system for protein using the aptamers in sandwich manner. Biosens. Bioelectron. 20:2168-2172.

5. Zhao, W., W. Chiuman, M.A. Brook, and Y. Li. 2007. Simple and rapid colorimetric biosensors based on DNA aptamer and noncrosslinking gold nanoparticle aggregation. ChemBioChem 8:727731.

6. Liu, J. and Y. Lu. 2005. Fast colorimetric sensing of adenosine and cocaine based on a general sensor design involving aptamers and nanoparticles. Angew. Chem. Int. Ed. Engl. 45:90-94.

7. Stojanovic, M.N., P. de Prada, and D.W. Landry. 2001. Aptamer-based folding fluorescent sensor for cocaine. J. Am. Chem. Soc. 123:4928-4931.

8. Tombelli, S., M. Minunni, E. Luzi, and M. Mascini. 2005. Aptamer-based biosensors for the detection of HIV-1 Tat protein. Bioelectrochemistry 67:135-141.

9. Bini, A., M. Minunni, S. Tombelli, S. Centi, and M. Mascini. 2007. Analytical performances of aptamer-based sensing for thrombin detection. Anal. Chem. 79:3016-3019.

10. Xiang, Y., Z. Wang, H. Xing, N.Y. Wong, and Y. Lu. 2010. Label-free fluorescent functional DNA sensors using unmodified DNA: a vacant site approach. Anal. Chem. 82:4122-4129.

11. Tuerk, C. and L. Gold. 1990. Systematic evolution of ligands by exponential enrichment: RNA ligands to bacteriophage T4 DNA polymerase. Science 249:505-510.

12. Bock, L.C., L.C. Griffin, J.A. Latham, E.H. Vermaas, and J.J. Toole. 1992. Selection of single-stranded DNA molecules that bind and inhibit human thrombin. Nature 355:564-566.

13. Travascio, P., Y. Li, and D. Sen. 1998. DNA-enhanced peroxidase activity of a DNA-aptamer-hemin complex. Chem. Biol. 5:505517
14. Pelossof, G., R. Tel-Vered, J. Elbaz, and I. Willner. 2010. Amplified biosensing using the horseradish peroxidase-mimicking DNAzyme as an electrocatalyst. Anal. Chem. 82:4396-4402.

15. Liu, J. and Y. Lu. 2006. Preparation of aptamerlinked gold nanoparticle purple aggregates for colorimetric sensing of analytes. Nat. Protoc. 1:246-252

16. Wu, Z.S., H. Lu, X. Liu, R. Hu, H. Zhou, G. Shen, and R.Q. Yu. 2010. Inhibitory effect of target binding on hairpin aptamer sticky-end pairinginduced gold nanoparticle assembly for light-up colorimetric protein assay. Anal. Chem. 82:38903898.

17. Ogasawara, D., N.S. Hachiya, K. Kaneko, K. Sode, and K. Ikebukuro. 2009. Detection system based on the conformational change in an aptamer and its application to simple bound/free separation. Biosens. Bioelectron. 24:1372-1376.

18. Chiuman, W. and Y. Li. 2007. Simple fluorescent sensors engineered with catalytic DNA 'MgZ' based on a non-classic allosteric design. PLoS ONE 2:e1224.

19. Huizenga, D.E. and J.W. Szostak. 1995. A DNA aptamer that binds adenosine and ATP. Biochemistry 34:656-665.

20. Knight, C.G., M. Platt, W. Rowe, D.C. Wedge, F. Khan, P.J. Day, A. McShea, J. Knowles, and D.B. Kell. 2009. Array-based evolution of DNA aptamers allows modelling of an explicit sequence-fitness landscape. Nucleic Acids Res. 37:e6.

21. Markham, N.R. and M. Zuker. 2008. UNAFold: software for nucleic acid folding and hybridization. Methods Mol. Biol. 453:3-31.

22. Teller, C., S. Shimron, and I. Willner. 2009 Aptamer-DNAzyme hairpins for amplified biosensing. Anal. Chem. 81:9114-9119.

23. Harada, K. and A.D. Frankel. 1995. Identification of two novel arginine binding DNAs. EMBO J. 14:5798-5811.

24. Mhadhbi, H., S. Benrejeb, and A. Martel. 2005 Studies on the affinity chromatography purification of anti-patulin polyclonal antibodies by enzyme linked immunosorbent assay and electrophoresis. Food Addit. Contam. 22:1243-1251.

25. de Champdoré, M., P. Bazzicalupo, L. De Napoli, D. Montesarchio, G. Di Fabio, I. Cocozza, A. Parracino, M. Rossi, and S. D'Auria. 2007. A new competitive fluorescence assay for the detection of patulin toxin. Anal. Chem. 79:751-757.

26. Jelinek, C.F., A.E. Pohland, and G.E. Wood. 1989. Worldwide occurrence of mycotoxins in foods and feeds--an update. J. Assoc. Off. Anal. Chem. 72:223-230.

27. Welke, J.E., M. Hoeltz, H.A. Dottori, and I.B. Noll. 2009. Quantitative analysis of patulin in apple juice by thin-layer chromatography using a charge coupled device detector. Food Addit Contam Part A Chem Anal Control Expo Risk Assess. 26:754-758.

28. Watanabe, M. and H. Shimizu. 2005. Detection of patulin in apple juices marketed in the Tohoku district, Japan. J. Food Prot. 68:610-612.

Received 10 November 2015; accepted 11 March 2016

Address correspondence to Yasuyuki Tomita, Central Laboratories for Key Technologies, KIRIN Company, Ltd., 1-13-5 Fukuura, Kanazawa-ku, Yokohama, Kanagawa 236-0004, Japan. E-mail: Yasuyuki_Tomita@kirin.co.jp

To purchase reprints of this article, contact: biotechniques@fosterprinting.com 